\title{
Research and Application of Multi-dimensional Analysis and Graphical Technology in Business Intelligence
}

\author{
Shan GAN \\ Electric Power Research Institute of Guangdong Power Grid Corporation, Guangzhou, Guangdong, 510080, \\ China
}

Haoquan DU

BaiZi Business Intelligence Information Technology Co,Ltd, Suzhou 215000, China

\begin{abstract}
In a Gartner survey on CIO technology priorities conducted last year, 55 percent of CIOs cited big data and analytics as the technologies they thought were most likely to be disruptive.(1)This trend has continued into 2014 and many businesses, including listed companies, are looking to information as the next frontier for competitive differentiation. Data has been critical to building market advantage for a long time, but until recently, most listed companies focused almost exclusively on player value databases to guide differentiation strategies. Organizations no longer have this luxury. Many markets face new competition as more neighboring jurisdictions allow gambling, and the emerging generation of patrons has shown a strong preference for a more diverse experience that includes much less time on the casino floor and more time in upgraded accommodations, spas, show theaters, full service restaurants, poolside, and other attractions. These aspects of the experience can still drive high margins, some arguably even higher than technology; but achieving this result requires harnessing data sources from many more departments, several of which often live in disparate systems and do not necessarily capture a guest's every transaction. In attempting to leverage all of this data, today's listed businesses are in danger of being overwhelmed by the sheer volume of information without a defined plan for how and where to utilize it [5]. These data through a good visual analysis, help the hypothetical conclusion put forward by the validation, guide the decisions of its new foresee and more efficient.
\end{abstract}

KEYWORD: business intelligence; data warehouse; datamart; mondrian; OLAP; dashboard

\section{INTRODUCTION}

In today's technology industry, collecting and analyzing real-time data can help properties to improve operations and customer service. However, many companies experience technology-related challenges that prevent them from fully utilizing the data that is aggregated. For example, at most properties the IT department controls business intelligence (BI) and analytics tools out of necessity because the tools are too complex for the average employee to use effectively. This means reporting and analysis is limited and often conducted only at the recommendation of c-level decision-makers. Another frequent roadblock is the lack of integration. With disparate systems, it is difficult to obtain a unified view across the business, meaning that data analysis is often segmented and lacking in visibility. Employees make decisions in isolation without consideration for how the actions of other departments might impact their results, simply because they do not have access to a single version of the truth.

With these challenges in mind, technology vendors are developing new, innovative BI applications designed to help technology companies maximize the full potential of their data. BI and analytics tools are becoming less complex and increasingly user friendly, with critical information displayed in easily consumable charts, graphs and dashboards. This enables technology properties to provide more users with access to these applications, eliminating the restriction of reporting and analysis capabilities to just the IT department. Equipping employees across multiple business units with a user-friendly BI application changes its role from a resource for the few to a resource for the masses. Additionally, technology providers who offer a flexible, lightweight middle-ware component give listed companies the opportunity to integrate existing business applications with a BI platform. Creating a unified solution allows organizations to compile data 
from multiple sources, enabling more in-depth analysis that is based on property-wide visibility.

\section{BI PRESENTATION LAYER TECHNOLOGY}

With the development of the peripheral technology, especially in the aesthetics of the customer for data presentation layer technology, ease of use, the pursuit of efficiency, prompted the front presentation layer development of business intelligence technology.

When effective analytics initiatives are implemented using a BI application that is both user friendly and integrated across business systems, technology properties can realize a multitude of benefits. By delivering information to employees when and where it is needed, properties can increase productivity by reducing the amount of time users spend looking for data. This helps to prevent errors and allows employees to spend time on more valueadd tasks. Access to critical information also enables faster response times across many departments. Further, analysis of this information can help organizations to pinpoint issues or take a proactive approach by foreseeing challenges before they arise.

But many powerful presentation layer technology is relatively dispersed and independent, unable to meet the overall needs of customers, while the mature Pentaho BI tool solved this problem nicely, not only incorporates many powerful and efficient tool in front presentation, but also prepared the interfaces for the future presentation layer technology's update and expand, truly realized progress with the time.

\section{IMPLEMENTATION OF PRESENTATION LAYER}

\subsection{Establish Data Warehouse and Preparation}

A data warehouse is a electronic storage of an Organization's historical data for the purpose of reporting, analysis and data mining or knowledge discovery.

Other than that a data warehouse can also be used for the purpose of data integration, master data management etc.

The structure of the data warehouse is to face multi-dimensional analysis, and is organized on subject-oriented way; you can use a star schema and snowflake model to implement multidimensional data relationships, dimensional model that contains the dimension and fact tables. Fact table stores a different transaction measurement and dimension tables' foreign keys. Dimensional model is not to obtain a high degree of standardization, but in order to facilitate easier, faster data retrieval. A fact can be quantified (or measured). The fact can usually (but not always) be aggregated. A dimension is used to define the measures. This allows analysis of business conditions from different angles, is given below an example of a sales analysis model.

\subsection{OLAP Design and Data Analytical}

OLAP (online analytical processing) is computer processing that enables a user to easily and selectively extract and view data from different points of view. OLAP data is stored in a multidimensional database. Whereas a relational database can be thought of as two-dimensional, a multidimensional database considers each data attribute (such as product, geographic sales region, and time period) as a separate "dimension." OLAP software can locate the intersection of dimensions (all products sold in the Eastern region above a certain price during a certain time period) and display them. Attributes such as time periods can be broken down into sub attributes.

\subsubsection{Star Schema}

This schema is used in data warehouse models where one centralized fact table references number of dimension tables so as the keys (primary key) from all the dimension tables flow into the fact table (as foreign key) where measures are stored. This entity-relationship diagram looks like a star, hence the name. As shown in figure 1:

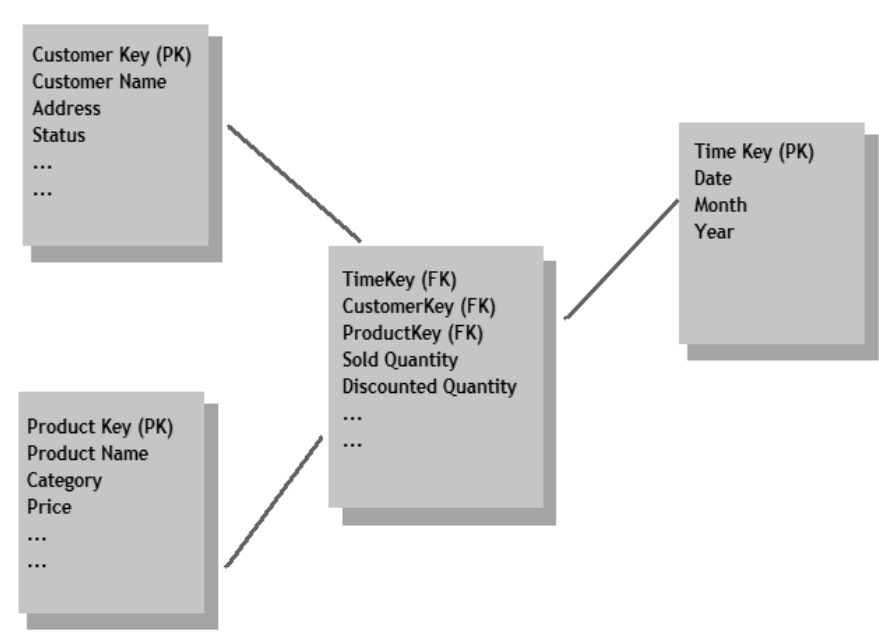

Figure1. Star Schema Model

Consider a fact table that stores sales quantity for each product and customer on a certain time. Sales quantity will be the measure here and keys from customer, product and time dimension tables will flow into the fact table.

\subsubsection{Snow-flake Schema}

This is another logical arrangement of tables in dimensional modeling where a centralized fact table references number of other dimension tables; 
however, those dimension tables are further normalized into multiple related tables.

Consider a fact table that stores sales quantity for each product and customer on a certain time. Sales quantity will be the measure here and keys from customer, product and time dimension tables will flow into the fact table. Additionally all the products can be further grouped under different product families stored in a different table so that primary key of product family tables also goes into the product table as a foreign key. Such construct will be called a snow-flake schema as product table is further snow-flaked into product family. As shown in figure 2:

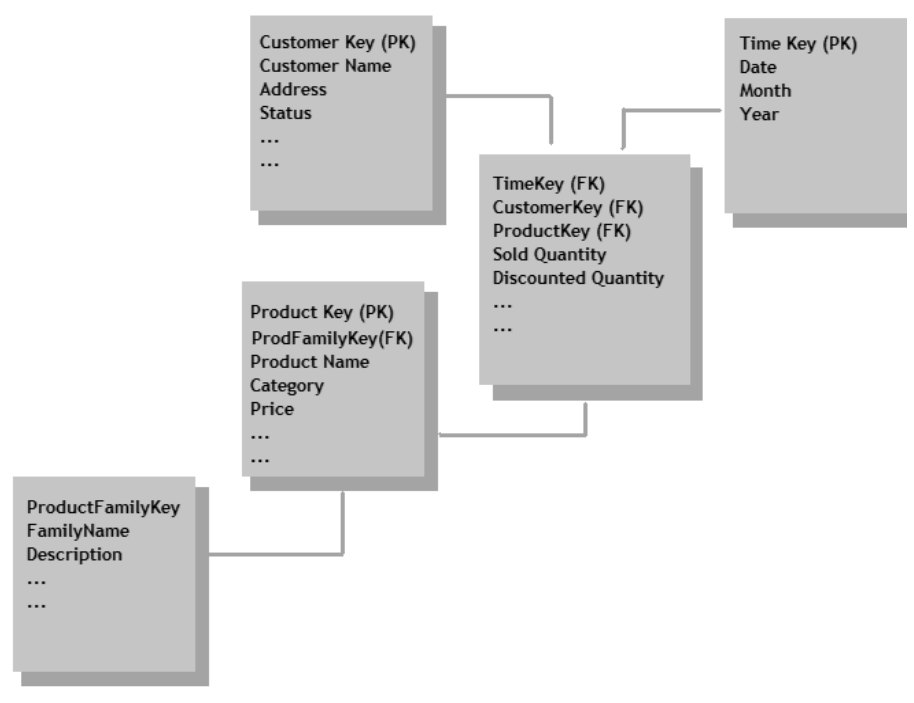

Figure2. Snow-flake Schema Model

\subsubsection{Fact tables}

Fact tables record measurements or metrics for a specific event. Fact tables generally consist of numeric values, and foreign keys to dimensional data where descriptive information is kept[3]. Fact tables are designed to a low level of uniform detail (referred to as "granularity" or "grain"), meaning facts can record events at a very atomic level. This can result in the accumulation of a large number of records in a fact table over time. Fact tables are defined as one of three types:

Transaction fact tables record facts about a specific event (e.g., sales events)

Snapshot fact tables record facts at a given point in time (e.g., account details at month end)

Accumulating snapshot tables record aggregate facts at a given point in time (e.g., total month-todate sales for a product)

Fact tables are generally assigned a surrogate key to ensure each row can be uniquely identified.

\subsection{Report Presentation}

In a database, report is mainly used for data grouping, calculation, aggregation and printouts. Report is one of the database objects. You can print out the formatted data. Database's print out is implemented by the report, using the report, the user can simply and easily print out complex information in a database. Reports are different from tables, it does not contain data, its data from base tables, queries, and SQL statements, but when running the information collected. Reports operation provides three kinds of views: Design view, print preview, the layout preview. It provides various different file format for the report export.

Report menu and system menu separation makes the main interface more simple and easier operation. Report elements editing area: report's major editing area, in the manner of display brief information subregions. And to provide for the report element to add, delete, modify, methods of operation functions. The following technical description uses reporting tool "Pentaho Report". The designer can be used in conjunction with Pentaho's BI platform and reporting system, it is based on open source JFreeReport technology.

JFreeReport API is a report writing foundation framework, its structure is similar to MSVC of Document-View (document view model). To make JFreeReport more universal, its internal use as a mechanism to hold the form TableModel report data. When reusing JFreeReport production databaseoriented reports, developers must obtain their own data from the database and populate the instance to be able to use the TableModel JFreeReport rendering, generate reports. JFreeReport just draw a higher level of abstraction of the basic tools of the report, use JFreeReport in the project, the programmer must also write data acquisition, such as code generation TableModel instance, while the report as a platform-level services, we may need to write complex report designer, interpreter and other user tools.

Given Pentaho has completed the above work, and open source code of their products, therefore Pentaho will be the basis for Report Designer and is integrated into our MIS system to implement the report service, this is a low cost, high speed, high product quality solution.

\subsubsection{Pentaho Reporting System Composition}

Pentaho Reporting System consists of three parts: Report interpreter (the kernel), Report Designer, Report publishing platform. Report developer using the Report Designer to generate a report template in the Report Designer, report developer can do database query generation, report layout, report preview, etc. Finally, you can publish reports to a report publishing platform; After the reports are published, report users can select a report on the platform, they can generate a report file in PDF, RTF, Excel and other formats; statements interpreter uses JFreeReport report generation tool in the designer directly include the interpreter; and the 
report publishing platform is actually a simplified configuration of Pentaho BI, Pentaho BI actually use internal Pentaho Sequence Flow and Pentaho Report Compoent components and the interpreter indirectly to interpret and generate the report [6].

\subsubsection{Pentaho Report Template and Interpreter}

Pentaho Report Designer generated report template file to report as $\mathrm{xml}$ file extension, a text editor to open the report template file can be found in these files included three parts, namely: the definition of a data source and data query, reporting within embedded data processing (function), report format information. Xml tag report format which is basically a report on JFreeReport extension xml meta tags and packaging.

The report will be based on the definition of the interpreter report template file data acquisition, data processing, in line with the last generation TableModel instance JFreeReport requirements, according to the definitions section of the format tag information generated JFreeReport reports show finally started JFreeReport engine to generate a variety of formats reports.

Through the above analysis it can be concluded, Pentaho Report accomplished custom user data queries, custom reports internal data processing, and with the powerful JFreeReport report presentation capability, it achieved a complete reporting, generating, publishing systems [6].

\subsection{Dashboard Presentation}

With the development of BI Dashboard technology has also progressed significantly, many independent Dashboard tools have emerged as ccchart, ecchart, googlechart, openflashchart, fusionchat. But these tools are relatively independent, can't be integrated, Pentaho rely on third-party-seamless-integration, the dashboard can be well integrated into their platform for the development of BI tools to make a leap of step also makes business intelligence more perfect, more welcomed by customers. As shown in figure 3:

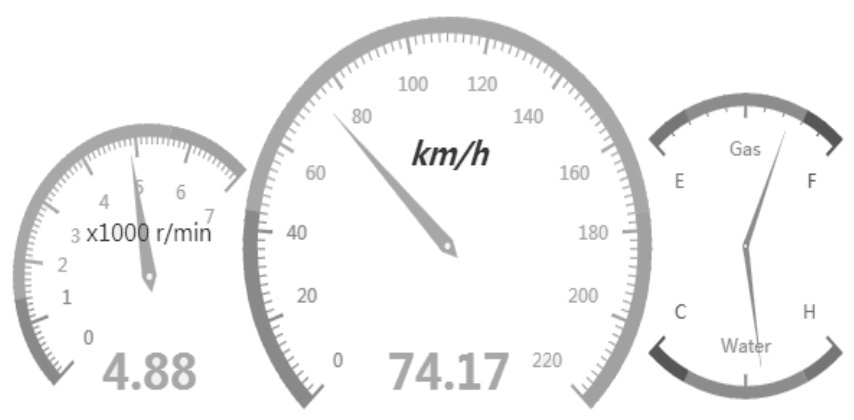

Figure3. Dashboard Presentation

\section{APPLICATIONS}

The current front-end business intelligence system to show a direct interaction with the user is a realization of the other browsers and operating data analysis, visualization, interactive applications; it is for decision-makers to get the basis for scientific data analysis, decision support. Scientific decision making is very important. Therefore, for the enterprise data analysts and IT researchers, conduct business intelligence data visualization work, for enhancing organizational decision making, integration and optimization of enterprise information resources and services, and improve the efficiency of decision-makers, etc., has significant theoretical and practical significance.

\section{CONCLUSION}

After the enterprise established BI visualization platform, decision making become easy and convenient, users can easily and timely analysis of business problems to extract the key factors that help corporate decision-making and business users to identify gaps between the actual situation, in-depth analysis of each specific Details of the situation in the field. With BI software companies have acquired BI visual presentation tools, and expand their capabilities to show the tools provided by the platform, making the visual presentation tools to further enhance application capabilities, Pentaho's constantly enhance its scalability, integration services platform escalating series of phenomena have fully showed a significant position in the BI front-end display technology system framework.

\section{REFERENCES}

[1] Shan Xiao. Visual information analysis. East China Normal University, 2010(in Chinese)

[2] Wei Chen. Data Business Intelligence in the visualization. Hefei University of Technology, 2010(in Chinese)

[3] Jing Li. Research and Application of OLAP-based data mining system, Shanghai University, 2003(in Chinese)

[4] Rongxin Chen, Yong Gang Fu, Weibin Chen. based Pentaho BI system. Computer Engineering and Design, 2008,09 phase (in Chinese)

[5] Bernard Ellis, Turning Big Data into Actionable Data,Vice President of Industry Strategy,2015(in USA)

[6] Cheng Gan. Integration of Pentaho report designer in the project. Zhengzhou institute of light industry 2009(in Chinese) 